\title{
DIRECTED EPILEPTIC NETWORK FROM SCALP AND INTRACRANIAL EEG OF EPILEPTIC PATIENTS
}

\author{
L. Amini ${ }^{1,2}$, C. Jutten ${ }^{1}$, S. Achard ${ }^{1}$, O. David ${ }^{3}$, H. Soltanian-Zadeh ${ }^{2,4}$, G.A. Hossein-Zadeh ${ }^{2}$, P. Kahane ${ }^{3}$, L. Minotti ${ }^{3}$, L. Vercueil ${ }^{3}$ \\ 1- GIPSA-LAB, Grenoble INP, Domaine universitaire- BP 46, F-38402 Grenoble Cedex, France. \\ 2- Control and Intelligent Processing Center of Excellence (CIPCE), University of Tehran, Tehran, Iran. \\ 3- Neurology Department and GIN U836 INSERM, CHUG, Grenoble, France. \\ 4- Radiology Image Analysis Laboratory, Henry Ford Health System, Detroit, MI 48202, USA.
}

\begin{abstract}
We proposed recently the computation of epileptic connectivity graphs based on wavelet correlation coefficients between EEG signals. The suspected epileptiform electrodes are recognized using the clustering of the topological properties of the graph that can be useful for pre-surgical studies. Here, we present a method for comparing epileptic networks estimated from scalp and intracranial EEG (IEEG) in partial epilepsy patients. The results are presented for a patient with left temporal epilepsy. Good spatial correspondence between the IEEG and the scalp EEG epileptic graphs is obtained. These results are consistent with the patient's clinical diagnosis.
\end{abstract}

\section{INTRODUCTION}

Drug resistant epileptic patients are recommended to undergo resective surgery. The pre-surgery evaluations are supposed to result in the localization of the presumed source of seizures and thus to optimally determine surgical candidate regions. There are already methods for epileptic neuronal source localization $[1,2]$, such as inverse problem methods $[3,4]$ and network analysis $[5,6]$. The question of identifying epileptic brain structures and their inter-connections remains open. The problem of characterizing structures with leading role in the epileptic activities is also remained unsolved. Here, we try to take step toward answering such questions. To this end we proposed a method based on the connectivity analysis for identifying epileptic sources and their primary and secondary inter-relationships.

Proposed connectivity measures in the literature can be divided into linear and non-linear methods. These two categories are compared in [5]. One problem of the linear methods like coherence function from the fast Fourier transform is characterized by high bias and variance. Filtering signals in frequency bands has suggested to address this problem

We gratefully acknowledge Ippeita Dan for providing the 10/20, 10/10, and 10/5 systems MNI coordinates of the scalp EEG.
[7, 8, 9]. Whitcher et al. [7] proposed the MODWT [10] correlation coefficients as a connectivity measure. Ansari et al. [9] also proposed the square of correlation coefficients between EEG signals filtered in narrow and overlapping frequency bands. The authors compared this measure with a standard estimator based on the coherence function showing better statistical performances (bias and mean squared error). Nonlinear methods can measure the possible nonlinear associations. Wendling et al. [5] presented the connectivity graph based on nonlinear regression during the transition from pre-ictal to seizure activity. The authors computed nonlinear correlation coefficient between each IEEG channel pair for periods of 10 seconds for each state (preictal period, ictal period, after termination of seizure). The nonlinear measure varied along these states. However, they showed that frequency-independent nonlinear methods like nonlinear regression, and methods using averaging over the frequency can not recognize the associations highly localized in frequency.

We used the MODWT correlation coefficients measure [7] with slightly improvement for our application. This measure does not have strong bias and variance as described above. Moreover, the wavelet coefficients have the same time sample size as input signal, since there is no decimation (of course the bordering effect should be considered). This property provides efficient time-frequency presentation of EEG signals. Time-frequency information of epileptiform and non-epileptiform time intervals from scalp EEG and IEEG signals is integrated into a differential connectivity graph $[11,12]$, discriminating connections between epileptic and non-epileptic states. These differential connections are chosen by a statistical test over the wavelet correlations of all of epileptiform and non-epileptiform time intervals to increase the robustness of the outcome. Extracted features from the differential graph are used to localize the suggested epileptic electrodes. The time causality between these electrodes is studied to identify the leading brain regions during the epileptic activity. Source localiza- 
tion by the scalp EEG graph analysis is to determine the electrodes close to the epileptic sources. This information as well as other physiologically related knowledge like MRI, fMRI, seizure semiology may be useful for the IEEG implantation planning. Furthermore, proposed leading epileptic brain tissues suggested by the IEEG graph analysis as well as other clinical knowledge can be helpful for resection surgical planning.

The paper is organized as follows. In Section 2, we describe the data protocol, and computing the directed epileptic graphs (DEG) computation. Section 3 is devoted to experimental results of the method from the scalp EEG and the IEEG signals. The DEG results and standard clinical evaluation are also compared. Concluding remarks are presented in Section 4.

\section{MATERIAL AND METHODS}

\subsection{Data}

The IEEG recordings were obtained from a 31 year old male with left temporal lobe epilepsy. He had the first seizure at the age of seven years. The standard clinical diagnosis using his scalp EEG showed an interictal spiking activity involved not only in the left temporal lobe, but also in the basal frontal and insular cortex. The patient underwent evaluation for resection surgery with the IEEG recordings [13]. $\mathrm{He}$ is seizure free after left temporal lobectomy surgery.

Eleven to fifteen semi-rigid multi-lead intracerebral electrodes with $0.8 \mathrm{~mm}$ diameter were bilaterally implanted in suspected seizure origins based on clinical considerations. The multi-lead electrodes (Dixi, Besançon, France) include $5,10,15$ or 18 leads evenly spaced every $1.5 \mathrm{~mm}$. Each lead has $2 \mathrm{~mm}$ length. The IEEG were recorded with an audiovideo-EEG monitoring system (Micromed, Treviso, Italy) with a maximum of 128 depth-EEG channels and digitized at $512 \mathrm{~Hz}$. The electrode leads were recognized on the patient's implantation scheme (part (a) of Fig. 1), and localized in the MNI atlas. Bipolar derivations were considered between adjacent leads within each electrode. For simplicity, these derivations are shown as $e_{i}$ instead of $e_{i+1}-e_{i}$. Scalp EEG recordings were recorded with 18 bipolar electrodes (according to the 10/20 standard (part (b) of Fig. 1) ). Signals were digitalized with a sampling rate of $256 \mathrm{~Hz}$ (Micromed). The MNI 10/20 system coordinates [14] is used for scalp EEG graph presentation. The bipolar derivations are shown as $e_{i} e_{j}(i \neq j)$ instead of $e_{j}-e_{i}$. Here, we focused on bipolar derivations in both scalp and intracranial EEG recordings. The bipolar derivation may provide more correctly measure differences of potentials between two recording electrodes [15].

\subsection{Directed epileptic graph (DEG) computation}

Here, we explain the different steps of DEG computation approach. DEG is a directed graph between suspected epileptic sources.

- Interictal epileptiform discharges (IED) and nonIED detection: The IED signals are detected manually by the expert neurologist. The start and end of IED signals is marked as IED labels. The non-IED labels are the time intervals without IED signals.

- Wavelet transform: Wavelet transform is chosen due to its good advantages over Fourier transform techniques for the EEG analysis [16]. The MODWT [10] is applied on the EEG data for two reasons: 1) to remove the low frequency trends and noise; 2) to compute the connectivity graph in different frequency bands. The IED signals of different patients and different IED types have different frequency distributions. The related frequency bands can be selected through computation of the connectivity graph for all of the scales in the wavelet domain. The scale giving the maximum number of connections is chosen in this study. However more than one frequency band (frequency bands giving considerable number of connections) can be studied.

- Differential connectivity graph (DCG) computation [11, 12]: The MODWT coefficients in selected frequency band is segmented as IED (non-IED) segments due to IED (non-IED) time intervals (labels).

We assume $N$-dimensional stationary IED and nonIED segments as $S_{m^{l}}^{l}[k]=\left[\mathbf{s}_{1}[k] \ldots \mathbf{s}_{N}[k]\right], \mathbf{s}_{1}, \ldots$, $\mathbf{s}_{N}$ are the columns of matrix $S . m^{l}=1, \ldots, N^{l}$, $k=1, \ldots, T\left(m^{l}\right) . N^{l}$ is the number of IED (nonIED) labels. $T\left(m^{l}\right)$ is the length of each IED (nonIED) segment.

The MODWT lagged correlation coefficients [7, 9] $\left(\hat{\rho}_{m^{l}}^{l}\left(\mathbf{s}_{i}, \mathbf{s}_{j}, \tau^{l}[k]\right),(i, j) \in\{1, \ldots, N\}, i \neq j\right)$ is estimated for each channel pair and IED (non-IED) segment in terms of different time lags $\left(\tau^{l}\right)$ :

$$
-10 \log _{10}\left(T\left(m^{l}\right) / 2\right) \leq \tau^{l}[k] \leq 10 \log _{10}\left(T\left(m^{l}\right) / 2\right)
$$

$\tau^{l}[k]$ is a function of $l$ and $k$, since $\tau$ of different IED (non-IED) labels can be different. $T\left(m^{l}\right)$ is the length of $S_{m^{l}}^{l}[k]$ or the length of each IED (non-IED) time interval. The maximum of this correlation coefficient $\left(\hat{\rho}_{m^{l}}^{\max }\right.$ ) over $\tau^{l}[k]$ is considered as the similarity measure. Next, a multiple hypothesis t-test $[17,18]$ is applied between the wavelet correlations $\left(\hat{\rho}_{m^{l}}^{\max }\right)$ of the IED and non-IED labels to distinguish the connections with different behaviors (wavelet correlations) 
in IED and non-IED segments using the following hypothesis:

$$
\begin{cases}H_{0}^{t}: & \mu_{t}^{1}=\mu_{t}^{2} \\ H_{1}^{t}: & \mu_{t}^{1} \neq \mu_{t}^{2}\end{cases}
$$

where $t=1, \ldots, N_{t}, N_{t}$ is the number possible connections, and $\mu^{l}$ is the mean of IED $(l=1)$ and non-IED $(l=2)$ wavelet correlations. The multiple testing procedure depends on an $N_{t}$-variate (estimated) test statistic null distribution for defining the rejection connections and adjusted p-values. A DCG graph shows the connections whose wavelet correlations are significantly different between the IED and non-IED time intervals. T-values of multiple hypothesis t-test (2) can be positive or negative. The positive (negative) DCG graph is a subgraph of the DCG with the connections whose $t$-values are positive (negative). The positive (negative) t-value connections show the increase of wavelet correlations during the IED (non-IED) time intervals.

- Source localization: The topological properties of the positive and negative DCGs are calculated using local and global efficiencies (LE and GE) [19]. LE and GE are calculated for all of the nodes (channels) in positive and negative DCGs. The power $t$-values (the power $t$-values between the IED and the non-IED segments in the related frequency band) are also calculated [11]. The scalp EEG electrodes or the IEEG electrode leads are clustered by the k-means method using a five-dimensional feature space (GE, LE of the positive and negative t-value graphs, and the power $\mathrm{t}$-value). The within cluster sum of squares is minimized to divide the $N$ nodes (channels) in five-dimensional space in to three clusters. The number of clusters is experimentally set to three. The features are normalized and their values are between zero and one. Source cluster (cluster including the suspected epileptic electrodes) is labeled prior physiological knowledge. By visual inspection, the channel (bipolar) containing most important IED signals is chosen by expert neurologist. The cluster including this channel is labeled as source cluster. The epileptic graph is a subgraph of the DCG which contains the connections between the source cluster electrodes.

- Timed causality: In order to determine the directions of the epileptic graph connections, the timed causality relations between the epileptic candidate pair electrodes are calculated.

We assume $P$-dimensional non-stationary observations of the EEG in selected frequency band (MODWT coefficients) as $X[n]=\left[\mathbf{x}_{1}[n] \ldots \mathbf{x}_{P}[n]\right] ; n=$ $1, \ldots, L . \mathbf{x}_{1}, \ldots, \mathbf{x}_{P}$ are the columns of matrix $X . P$ and $L$ are the number of source cluster electrodes and the length of the original (non segmented) EEG signal (time sample), respectively. The wavelet correlation coefficient $\left(\hat{\rho}\left(\mathbf{x}_{i}, \mathbf{x}_{j}, \tau\right)\right)$ and the maximum wavelet correlation coefficient $\left(\hat{\rho}^{\max }\left(\mathbf{x}_{i}, \mathbf{x}_{j}\right)\right)$ for a given pair of channels $(i, j) \in\{1, \ldots, P\}, i \neq j$ are computed via (3) and (4), respectively:

$$
\begin{gathered}
\frac{\hat{\operatorname{Cov}}\left\{\mathbf{x}_{i}[k], \mathbf{x}_{j}[k+\tau]\right\}}{\sqrt{\hat{\operatorname{Var}}\left(\mathbf{x}_{i}[k]\right) \hat{\operatorname{Var}}\left(\mathbf{x}_{j}[k+\tau]\right)}} \\
\hat{\rho}^{\max }\left(\mathbf{x}_{i}, \mathbf{x}_{j}\right)=\max _{\tau} \hat{\rho}\left(\mathbf{x}_{i}, \mathbf{x}_{j}, \tau\right)
\end{gathered}
$$

where $-\operatorname{10log}_{10}(L / 2) \leq \tau \leq 10 \log _{10}(L / 2)$ is the sample lag. Cov and $\operatorname{Var}$ [7] are the estimated covariance and variance, respectively. $\hat{\rho}\left(\mathbf{x}_{i}, \mathbf{x}_{j}, \tau\right)$ has the following property:

$$
\hat{\rho}\left(\mathbf{x}_{i}, \mathbf{x}_{j}, \tau\right)=\hat{\rho}\left(\mathbf{x}_{j}, \mathbf{x}_{i},-\tau\right) .
$$

The $\tau$ corresponding to the maximum wavelet correlation coefficient $\left(\hat{\rho}^{\max }\left(\mathbf{x}_{i}, \mathbf{x}_{j}\right)\right)$ is defined as timed causality between $\mathbf{x}_{i}$ and $\mathbf{x}_{j}$, and denoted as $\tau^{\max }$. In theory, $\tau^{\max }$ is equal to zero for concurrent signals and negative (positive) if $\mathbf{x}_{i}\left(\mathbf{x}_{j}\right)$ causes $\mathbf{x}_{j}\left(\mathbf{x}_{i}\right)$. Due to the property of (5), the timed causality between $\mathbf{x}_{j}$ and $\mathbf{x}_{i}$ is negative (positive), if $\mathbf{x}_{j}\left(\mathbf{x}_{i}\right)$ causes $\mathbf{x}_{i}\left(\mathbf{x}_{j}\right)$. To end with, the timed causality $\left(\tau^{\max }\right.$ ) between $\mathbf{x}_{i}$ and $\mathbf{x}_{j}$ is as following:

$$
\begin{cases}0 & \mathbf{x}_{i} \text { and } \mathbf{x}_{j} \text { are time concurrent } \\ \text { negative } & \mathbf{x}_{i} \text { time causes } \mathbf{x}_{j} \\ \text { positive } & \mathbf{x}_{j} \text { time causes } \mathbf{x}_{i}\end{cases}
$$

The timed causality determines the direction of the connections in the epileptic graph and its absolute value defines the weights of the connections.

- In-degree and out-degree: A simple topological quantification of the nodes of the DEG can be obtained by in-degree and out-degree measures [20]. The indegree $d_{i}^{i n}$, and out-degree $d_{i}^{\text {out }}$ of a node $i$ is respectively the number of ingoing and outgoing edges incident to the node:

$$
d_{i}^{\text {in }}=\sum_{j \in P} c_{j i}, \quad d_{i}^{\text {out }}=\sum_{j \in P} c_{i j}
$$

where $P$ is the number of source cluster electrodes, $i, j=1, \ldots, P . \quad c_{i j}$ is the entry of the adjacency (or timed causality) matrix $C \in \mathbb{R}^{P \times P} . c_{i j(i<j)}$ is negative (positive) when there is an edge from $i$ to $j$ (from $j$ to $i$ ). The node with maximum out-degree and in-degree can be considered as source and target of DEG, respectively. Origin of IED source may rise from some brain tissues and then propagate to other regions. Due to this assumption, identification of source and target nodes of source cluster electrodes is important. 


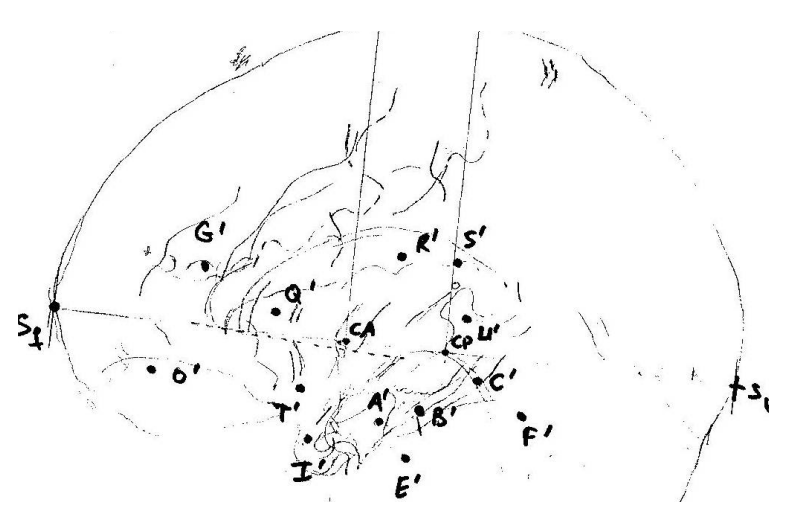

(a) IEEG electrodes'implantation scheme

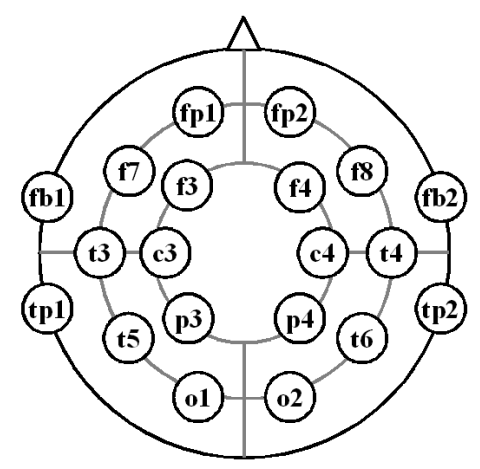

(b) 10/20 system of scalp EEG electrodes' placement

Fig. 1. (a) Implantation scheme of the IEEG electrodes (sagittal view); (b) 10/20 system of scalp EEG electrodes' placement.

\section{RESULTS AND DISCUSSION}

The proposed method is applied on five partial epilepsy patients' EEG data. Here, we report the results of the method in a left temporal lobe epilepsy EEG data (Scalp and intracranial EEG). The parameters of the method are summarized in Table 1. Parts (a-b) of Fig. 1 are the implantation scheme of the IEEG electrodes (sagittal view) and 10/20 system scalp EEG electrodes' placements, respectively. Fig. 2 illustrates the comparison of the directed epileptic graphs (DEG) from scalp and intracranial EEG (IEEG) superimposed on a 3D anatomical mesh. Parts (a-c) show the DEGs from axial, sagittal, and coronal views, respectively. This figure is presented to show the spatial relationship between the IEEG and the scalp EEG DEGs. Good spatial agreement between these graphs is observed. Parts (a-b) of Fig. 3 show the close view (zoom in on Fig. 2) of DEGs from the scalp EEG and IEEG, respectively. Solid (dashed) lines show positive (negative) t-value graph connections. Positive (negative) t-value connections illustrate the significant increase of the wavelet correlations during IED (non-IED) time intervals. The thickness of the lines is proportional to

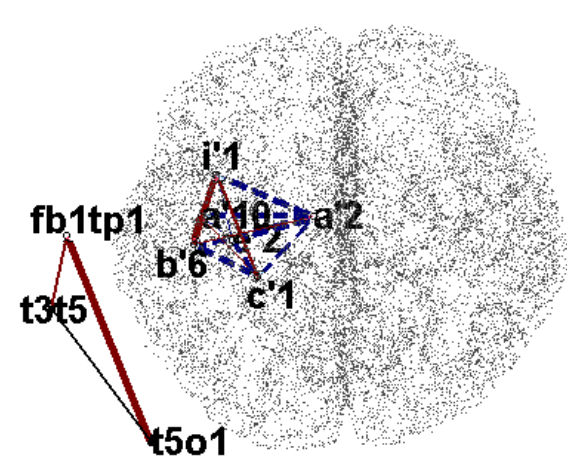

(a) Scalp EEG and IEEG, axial view

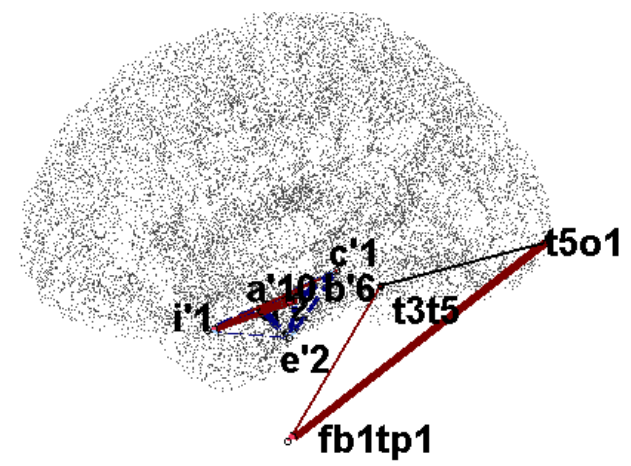

(b) Scalp EEG and IEEG, sagittal view

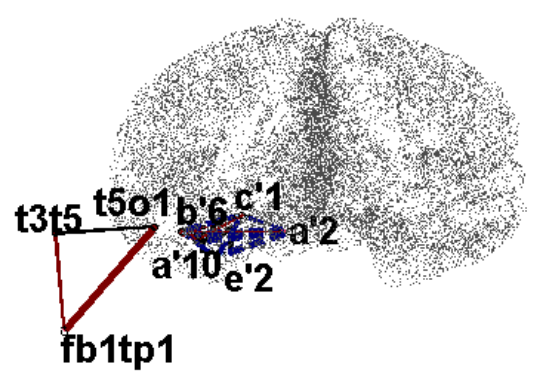

(c) Scalp EEG and IEEG, coronal view

Fig. 2. Comparison (spatial correspondence) of directed epileptic graph (DEG) from the scalp EEG and the IEEG superimposed on a 3D anatomical mesh. (a) Axial; (b) sagittal, and (c) coronal view. The positive (negative) t-value connections are depicted in solid (dashed) lines. The close views of scalp and IEEG DEGs are presented in Fig. 3.

the absolute values of $\tau^{\max }$ defined in the following of (5) in Section 2.2. $\tau^{\max }$ is the time delay related to the maximum wavelet correlation coefficient between long (more than one hour recording with sampling frequency equal to $512 \mathrm{~Hz}$ ) and noise free (wavelet coefficients of the related frequency band $(2-4 \mathrm{~Hz}$ ) are assumed to be noise free) signals [21]. Here we used the theoretical constraint (1) for calculating $\tau^{\max }$. Of course concerning the approximate max- 

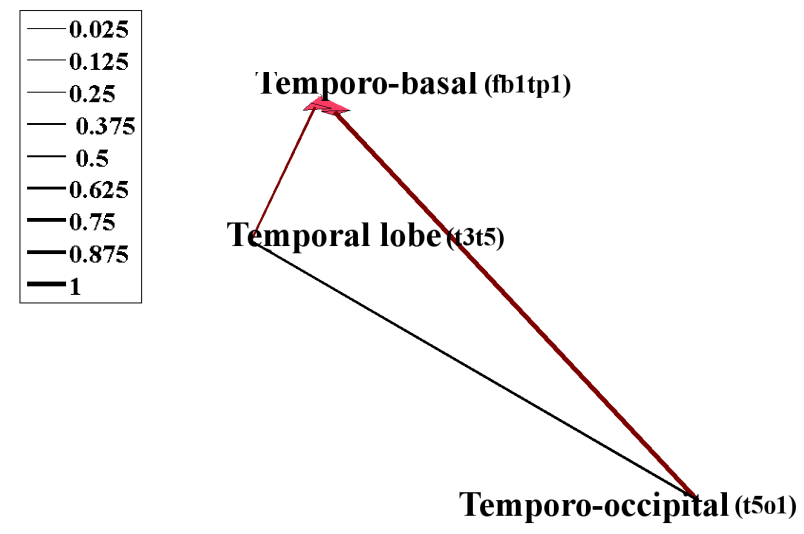

(a) Scalp EEG

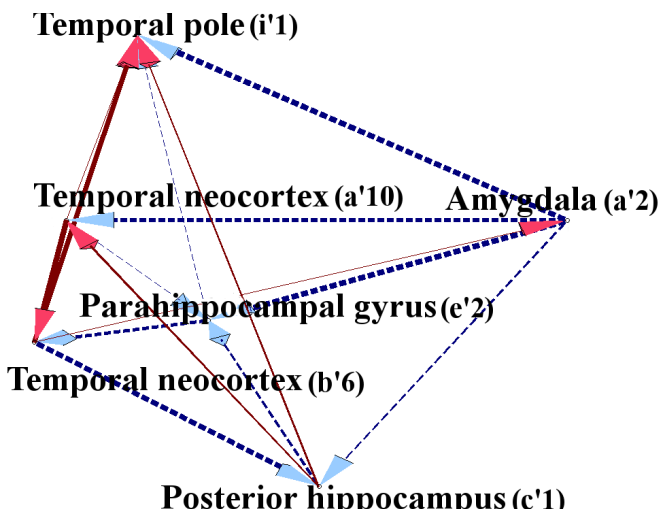

(b) IEEG

Fig. 3. DEG from (a) scalp EEG; and (b) IEEG. (a-b) are the close view (zoom in) of Fig.2. Positive (negative) tvalue graph connections show the significant increase of the wavelet correlations during IED (non-IED) time intervals. The thickness of the connections are proportional to the absolute values of $\tau^{\max }$ defined in the following of (5) in Section 2.2. The positive (negative) t-value connections are depicted in solid (dashed) lines. The connection without arrow in part (a) shows the time concurrency of the related nodes.

imum and minimum nerve conduction velocity and the distance between electrodes may provide more accurate physiological constraints [15]. However the connections are not necessarily direct which complicates the determination of these constraints. Here, the sign of $\tau^{\max }$ is used to define the direction of epileptic graph connections.

The visual inspection of interictal spiking on the scalp EEG and clinical data (seizure semilogy analysis) of the left temporal patient suggested the involvement of the left temporal lobe, with secondary propagation to basal frontal cortex through insular cortex. After the confirmation of presenting hypothesis by means of IEEG recording (interictal and ictal recordings), the patient underwent left temporal
Table 1. Parameters of the method. $N$ : number of the bipolar channels; $P$ : number of epileptic candidate electrodes (scalp EEG) or electrode contacts (IEEG); $L$ : length of the original (non segmented) EEG signal (hour); $N_{t}$ : number of possible connections, $N^{l}$ : number of IED $(l=1)$ and non$\operatorname{IED}(l=2)$ labels.

\begin{tabular}{lll}
\hline Parameter & Scalp & Intracranial \\
\hline$N$ & 18 & 31 \\
$P$ & 3 & 6 \\
$L$ (hour) & 1.27 & 1.20 \\
$N_{t}$ & 153 & 465 \\
$N^{1}$ & 133 & 411 \\
$N^{2}$ & 169 & 143 \\
IED frequency band (Hz) & $2-4$ & $2-4$ \\
Sampling rate (Hz) & 256 & 512 \\
Wavelet filter & $\mathrm{db} 4$ & $\mathrm{db} 4$ \\
Number of wavelet levels & 9 & 9 \\
False positive error & 0.05 & 0.05 \\
\hline
\end{tabular}

Table 2. In-degree and out-degree of the directed epileptic graph. T: temporal; TO: temporo-occipital; TB: temporobasal; TP: temporal pole; amyg: amygdala; TN: temporal neocortex; pHcG: parahippocampal gyrus; post Hc: posterior hippocampus; lb: lobe; rg: region.

\begin{tabular}{|c|c|c|c|}
\hline & Brain region (electrode) & in-degree & out-degree \\
\hline \multirow{3}{*}{$\begin{array}{l}\text { స్ } \\
\text { డ్ }\end{array}$} & $\mathrm{T}-\mathrm{lb}(t 3 t 5)$ & 0 & 1 \\
\hline & TO-rg $(t 5 o 1)$ & 0 & 1 \\
\hline & TB-rg $(f b 1 t p 1)$ & 2 & 0 \\
\hline \multirow{6}{*}{ 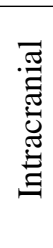 } & TP $\left(i^{\prime} 1\right)$ & 5 & 0 \\
\hline & $\operatorname{amyg}\left(a^{\prime} 2\right)$ & 1 & 4 \\
\hline & $\mathrm{TN}\left(a^{\prime} 10\right)$ & 2 & 3 \\
\hline & $\mathrm{pHcG}\left(e^{\prime} 2\right)$ & 3 & 2 \\
\hline & $\mathrm{TN}\left(b^{\prime} 6\right)$ & 2 & 3 \\
\hline & post $\mathrm{Hc}\left(c^{\prime} 1\right)$ & 2 & 3 \\
\hline
\end{tabular}

lobectomy surgery four years ago and he is seizure free. The resected region includes the IEEG electrodes $\left\{i^{\prime} a^{\prime} b^{\prime} e^{\prime} c^{\prime}\right\}$. In our scalp EEG analysis, temporal lobe ( $t 3 t 5)$, temporo-occipital ( $f b 1 t p 1)$, and temporo-basal regions $(t 5 o 1)$ are identified as suggested epileptic regions (part (a) of Fig. $3)$. The epileptic regions proposed by the IEEG analysis are temporal pole $\left(i^{\prime} 1\right)$, amygdala $\left(a^{\prime} 2\right)$, temporal neocortex ( $a^{\prime} 10$ and $\left.b^{\prime} 6\right)$, posterior hippocampus $\left(c^{\prime} 1\right)$, and parahippocampal gyrus $\left(e^{\prime} 2\right)$, which are in left temporal lobe. These results are consistent with the resected regions in the surgery. Successful patient's treatment may suggest good evaluation of the method's results.

The directions of the epileptic graph connections adds extra interpretable information about the source and target epileptiform zones. The in-degree and out-degree of DEGs from the scalp EEG and the IEEG are reported in Table 
2. According to the scalp DEG findings, temporal lobe $(t 3 t 5)$ and temporo-occipital region $(t 5 o 1)$ are time concurrent, and both of them are causing temporo-basal region ( $f b 1 t p 1$ ), but with different $\tau^{\max }$. $\tau^{\max }$ between temporal lobe and temporo-basal region $(3.9 \mathrm{msec})$ is less than $\tau^{\max }$ between temporo-occipital and temporo-basal regions $(7.8 \mathrm{msec})$. As can be seen in Table 2, temporo-basal region (target) has the maximum in-degree, and temporal lobe (source) have the maximum out-degree. Since the connection between temporal lobe $(t 3 t 5)$ and temporo-occipital region $(t 5 o 1)$ is time concurrent, this edge is not considered in degree computation. Temporal lobe $(\{t 3 t 5, t 5 o 1\}$, presumably $t 5$ ) and temporo-basal region could be primarily and secondarily involved by the epileptic discharges, respectively. In the intracranial DEG, amygdala (source) and temporal pole (target) have the maximum out-degree and in-degree, respectively (Table 2). It means that amygdala could contain the main epileptic source, while temporal pole might receive the secondary propagated IED signals. Such proposition would be very challenging to detect visually. The extracted information may be a step toward better understanding of the interactions between epileptic zones and therefore towards enhanced pre-surgical studies to restrict patients' resected regions while insuring the patients' treatment.

\section{CONCLUSION}

We studied the connectivity graph of suggested epileptic sources from the scalp and intracranial EEG of epileptic patients. We computed the connectivity graph using time and frequency properties of EEG signals. Using graph characterization, suspected leading epileptic electrodes (from the scalp EEG) and electrode contacts (from IEEG) can be suggested prior to the planning of the IEEG electrodes implantation and resection surgery, respectively. The future work is to concentrate on more accurate topological quantification of the directed epileptic graph to identify source and target brain regions. In other words, we can estimate the probability of resecting each suspected source brain tissues by means of quantifying if the epileptic zone rises (source) or receives (target) the IED signals.

\section{REFERENCES}

[1] J. S. Ebersole and S. Hawes-Ebersole, "Clinical Application of Dipole Models in the Localization of Epileptiform Activity," J Clin Neurophysiol, vol. 24, no. 2, pp. 120-129, Apr 2007.

[2] P. J. Uhlhaas and W. Singer, "Neural Synchrony in Brain Disorders: Relevance for Cognitive Dysfunctions and Pathophysiology," Neuron, vol. 52, no. 1, pp. 155-168, Oct 2006.

[3] Y. Zhang, W. van Drongelen, M. Kohrman, and B. He, “Threedimensional Brain Current Source Reconstruction From Intra-cranial ECoG Recordings," Neuroimage, vol. 42, no. 2, pp. 683-695, 2008.

[4] D. Zumsteg, A. Friedman, H. G. Wieser, and R. A. Wennberg, "Source Localization of Interictal Epileptiform Discharges: Com- parison of Three Different Techniques to Improve Signal to Noise Ratio," Clin Neurophysiol, vol. 117, no. 3, pp. 562-571, Mar 2006.

[5] F. Wendling, F. Bartolomei, and L. Senhadji, "Spatial Analysis of Intracerebral Electroencephalographic Signals in the Time and Frequency Domain: Identification of Epileptogenic Networks in Partial Epilepsy," Philos. Trans. R. Soc. A-Math. Phys. Eng. Sci., vol. 367, pp. 297-316, 2009.

[6] O. David, A. Wozniak, L. Minotti, and P. Kahane, "Preictal Shortterm Plasticity Induced by Intracerebral $1 \mathrm{~Hz}$ Stimulation," NeuroImage, vol. 39, no. 4, pp. 1633 - 1646, 2008.

[7] B. Whitcher, P. Guttorp, and D. Percival, "Wavelet Analysis of Covariance with Application to Atmospheric Time Series," J. Geophys. Res. - Atmospheres, vol. 105, pp. 14941-14962, 2000.

[8] F. Wendling, F. Bartolomei, J. J. Bellanger, J. Bourien, and P. Chauvel, "Epileptic Fast Intracerebral EEG Activity: Evidence for Spatial Decorrelation at Seizure Onset," Brain, vol. 126, no. Pt 6, pp. 14491459, Jun 2003.

[9] K. Ansari-Asl, J. J. Bellanger, F. Bartolomei, F. Wendling, and L. Senhadji, "Time-Frequency Characterization of Interdependencies in Nonstationary Signals: Application to Epileptic EEG," TBME, vol. 52, pp. 1218-26, 2005.

[10] D.B. Percival and A.T. Walden, "Wavelet Methods for Time Series Analysis," Cambridge, UK: Cambridge UP, 2000.

[11] L. Amini, S. Achard, C. Jutten, H. Soltanian-Zadeh, G. A. HosseinZadeh, O. David, and L. Vercueil, "Sparse Differential Connectivity Graph of Scalp EEG for Epileptic Patients," in ESANN2009, Bruges, Belgium, April 2009.

[12] L. Amini, C. Jutten S. Achard, G.A. Hossein-Zadeh, and H. Soltanian-Zadeh, "Connectivity Analysis of EEG Recordings for Epileptic Patients," in ICON X, Bodrum, Turkey, Sep. 1 - 5, 2008.

[13] P. Kahane, L. Minotti, D. Hoffmann, J. Lachaux, and P. Ryvlin, "Invasive EEG in the Definition of the Seizure Onset Zone: Depth Electrodes," Handbook of Clin. Neurophysiol. Pre-surgical assessment of the epilepsies with clinical neurophysiology and functional neuroimaging (Rosenow, F. and Liiders, H.O., eds.): Elsevier Science, 2004.

[14] V. Jurcak, D. Tsuzuki, and I. Dan, "10/20, 10/10, and 10/5 Systems Revisited: Their Validity as Relative Head-Surface-Based Positioning Systems," Neuroimage, vol. 34, no. 4, pp. 1600-1611, Feb 2007.

[15] P. L. Nunez and R. Srinivasan, "Electric Fields of the Brain: The Neurophysics of EEG”, Oxford University Press, Inc., 2006.

[16] M. Unser S. Sato S. J. Schiff, A. Aldroubi, "Fast Wavelet Transformation of EEG," Electroencephalogr Clin Neurophysiol., vol. 91, pp. 442-55, 1994.

[17] M. J. van der Laan, S. Dudoit, and K. S. Pollard, "Multiple Testing. Part II. Step-Down Procedures for Control of the Family-Wise Error Rate," Stat. Appl. Genet. Mol. Biol., vol. 3, 2004.

[18] K. S. Pollard, M. D. Birkner, M. J. van der Laan, and S. Dudoit, "Test Statistics Null Distributions in Multiple Testing: Simulation Studies and Applications to Genomics," Journal de la Société Française de Statistique., vol. 146, pp. 77-115, 2005.

[19] D. J. Watts and S. H. Strogatz, "Collective Dynamics of Small-world Networks," Nature, vol. 393, pp. 440-442, 1998.

[20] S. Boccaletti, V. Latora, Y. Moreno, M. Chavez, and D. U. Hwang, "Complex Networks: Structure and Dynamics," Physics Reports, vol. 424, no. 4-5, pp. 175-308, 2006.

[21] S. J. Schiff, P. So, T. Chang, R. E. Burke, and T. Sauer, "Detecting Dynamical Interdependence and Generalized Synchrony Through Mutual Prediction in a Neural Ensemble.," Phys Rev E Stat Phys Plasmas Fluids Relat Interdiscip Topics, vol. 54, no. 6, pp. 67086724, Dec 1996. 